\title{
SYMMETRIC FUNCTIONS, LEBESGUE MEASURABILITY, AND THE BAIRE PROPERTY
}

\author{
UDAYAN B. DARJI
}

(Communicated by Andrew M. Bruckner)

\begin{abstract}
In this paper, we generalize some results of Stein and Zygmund and of Evans and Larson concerning symmetric functions. In particular, we show that if $f$ is Lebesgue measurable or has the Baire property in the wide sense, then the set of symmetric points of $f$ is Lebesgue measurable or has the Baire property in the wide sense, respectively. We also give some examples that show that these results cannot be improved in a certain sense. Finally, we show that there are plenty of examples of functions that are both Lebesgue measurable and have the Baire property in the wide sense, yet the set of points where each of the functions is symmetric and discontinuous has the same cardinality as that of the continuum.
\end{abstract}

\section{INTRODUCTION}

A function $f: R \rightarrow R$ is said to be symmetric at $x \in R$ if

$$
\lim _{h \rightarrow 0} f(x+h)+f(x-h)-2 f(x)=0,
$$

and $f$ is symmetric if $f$ is symmetric at every $x \in R$.

In 1964 Stein and Zygmund in [8] showed that if $f: R \rightarrow R$ is Lebesgue measurable and $f$ is symmetric on a Lebesgue measurable set $M$, then $f$ is continuous a.e. on $M$. In 1984 Evans and Larson in [3] showed that if $f: R \rightarrow R$ has the Baire property in the wide sense and $f$ is symmetric on a set $M$ which has the Baire property in the wide sense, then the set of all points of $M$ where $f$ is discontinuous is of first category.

In this paper, we show that in the aforementioned theorems the additional hypothesis of $M$ being measurable or having the Baire property in the wide sense is not necessary. Denote by $S(f)$ the set of points where $f$ is symmetric and by $C(f)$ the set of points where $f$ is continuous. We show that if $f$ is Lebesgue measurable or has the Baire property in the wide sense, then $S(f) \backslash C(f)$ is of measure zero or first category, respectively. As corollaries, we

Received by the editors December 10,1991; presented at the 8th Auburn Miniconference on Real Analysis, March, 1991, sponsored by NSF.

1991 Mathematics Subject Classification. Primary 26A21, 26A15; Secondary 54C30, 54C50, $54 \mathrm{H} 05$.

Key words and phrases. Symmetric functions, the Baire property, Lusin sets, Sierpinski sets. 
get that if $f$ is Lebesgue measurable or has the Baire property in the wide sense, then $S(f)$ is Lebesgue measurable or has the Baire property in the wide sense, respectively.

In 1964 Neugebauer in [5] showed that if $f: R \rightarrow R$ is symmetric and Lebesgue measurable, then $f$ has to be of Baire class 1. In 1984 Evans and Larson in [3] showed that if $f: R \rightarrow R$ is symmetric and has the Baire property in the wide sense, then $f$ is of Baire class 1. It follows from the Evans-Larson theorem and the Neugebauer theorem that a symmetric function $f: R \rightarrow R$ is Lebesgue measurable iff it has the Baire property in the wide sense; however, this is not true if the function is not symmetric on the entire line as will be evident by Examples 8 and 9.

Lastly, we show that there are plenty of examples of functions which are simultaneously Lebesgue measurable and have the Baire property in the wide sense, yet the set of points where each of the functions is symmetric and discontinuous is uncountable.

Let us now state some definitions and background theorems.

Recall that a set $M \subseteq R$ has the Baire property in the wide sense, or for short $M \in B_{w}$, if it is the difference of an open set and a first category set. This is equivalent to saying that $M$ is the union of two sets, one of which is $G_{\delta}$ and the other of the first category. A set $M$ is categorically dense in some interval $I$ means that $M \cap J$ is second category for every interval $J \subseteq I$. Also recall that every second category set is categorically dense in some interval. A set $M \subseteq R$ has the Baire property in the restricted sense, or for short $M \in B_{r}$, means that $M$ has the Baire property in the wide sense relative to every perfect set $P$ (i.e., $M \cap P$ is $B_{w}$ relative to $P$ ). A set $M \subseteq R$ is always first category, or for short $M \in \mathrm{AFC}$, if every subset of $M$ has the Baire property in the restricted sense. A function $f: R \rightarrow R$ has the Baire property in the wide sense (the Baire property in the restricted sense) means that the preimage of every open set under $f$ has the Baire property in the wide sense (the Baire property in the restricted sense).

We will let $\mu(M), \mu^{*}(M)$, and $\mu_{*}(M)$ denote the Lebesgue measure of $M$, the outer Lebesgue measure of $M$, and the inner Lebesgue measure of $M$, respectively. Also recall that saying $M$ is Lebesgue measurable is equivalent to saying that for every perfect set $P$ with positive measure, there exists a perfect set $Q \subset P$ with positive measure such that $Q \subseteq M$ or $Q \subseteq M^{c}$. From this definition, it easily follows that if $M$ is a set with positive outer measure then there exists a perfect set $P$ with positive measure such that $M$ has full outer measure in $P$. A set $M \subseteq R$ is universally measurable, or for short $M \in U$, iff $M$ is measurable according to the completion of every nonatomic Borel measure on $R$. A set $M \subseteq R$ is $U_{0}$ iff every subset of $M$ is universally measurable. A function $f: R \rightarrow R$ is universally measurable means that the preimage of every open set under $f$ is universally measurable.

An uncountable set $M \subseteq R$ is a Lusin set (Sierpinski set) iff the intersection of $M$ with every first category set (measure zero set) is countable. Under the assumption of the continuum hypothesis, there are uncountable Lusin and Sierpinski sets. Also recall that the Lusin sets do not have the Baire property in the wide sense, yet they are $U_{0}$; the Sierpinski sets are nonmeasurable, yet they are AFC. Refer to [4] for more information about the Baire property, universal measurability, AFC sets, $U_{0}$ sets, Lusin sets, and Sierpinski sets. 
If $r \in R$ and each of $A$ and $B$ is a subset of the real line, then $r A=\{r x$ : $x \in A\}, r+A=\{r+x: x \in A\}$, and $A+B=\{x+y: x \in A$ and $y \in B\}$.

\section{MAIN RESUltS}

Theorem 1. If $f: R \rightarrow R$ has the Baire property in the wide sense, then $S(f) \backslash C(f)$ is of first category.

Proof. Suppose that $S(f) \backslash C(f)$ is second category. Then $S(f) \backslash C(f)$ is categorically dense in some closed interval $I$. We shall show that this situation is impossible by showing that $C(f)$ is actually dense in this $I$.

Suppose that $C(f)$ is not dense in $I$ and $I_{1}$ is a closed subinterval of $I$ containing no point of $C(f)$. Using the fact that $I_{1} \cap C(f)=\varnothing$ and the Baire category theorem, we may obtain a closed subinterval $I_{2} \subset I_{1}$ and a rational $\varepsilon>0$ such that $\operatorname{osc}(f, x)>\varepsilon$ for each $x \in I_{2}$. Here $\operatorname{osc}(f, x)$ denotes the oscillation of $f$ at $x$.

Now let $\left\{J_{k}\right\}$ be an enumeration of all open intervals of length $\varepsilon / 3$ having rational end points. There must be $k$ such that $f^{-1}\left(J_{k}\right) \cap I_{2}$ is second category. Since $f$ has the Baire property in the wide sense, $f^{-1}\left(J_{k}\right) \cap I_{2}$ contains a second category $G_{\delta}$ set, which, consequently, is residual in some closed interval $I_{3} \subseteq I_{2}$.

Let $J \equiv J_{k} \equiv(a, b)$ and $K \equiv R \backslash(a-2 \varepsilon / 3, b+2 \varepsilon / 3)$. Since osc $(f, x)>\varepsilon$ for each $x \in I_{3}, f^{-1}(K)$ is dense in $I_{3}$. We now make the following claim: For each closed interval $H \equiv[u, v] \subseteq I_{3}$, there is a closed subinterval $H^{*} \subseteq H$ such that $\left\{(s+t) / 2: s \in f^{-1}(J) \cap H\right.$ and $\left.t \in f^{-1}(K) \cap H\right\}$ contains a dense $G_{\delta}$ subset of $H^{*}$. To see this, let $p \in f^{-1}(K) \cap H$. Since $f^{-1}(J) \cap H$ contains a dense $G_{\delta}$ subset of $H$, it follows that the set $\left\{(s+p) / 2: s \in f^{-1}(J) \cap H\right\}$ contains a $G_{\delta}$ subset which is dense in $H^{*}=((u+p) / 2,(p+v) / 2)$.

For each natural number $j$, let $G_{j}=\left\{x \in I_{3}\right.$ : there is a $0<h<1 / j$ such that one of $x+h, x-h$ belongs to $f^{-1}(K)$ while the other belongs to $\left.f^{-1}(J)\right\}$. Each $G_{j}$ is residual in $I_{3}$. To see this, suppose that $I_{3} \backslash G_{j}$ is second category and let $H \subseteq I_{3}$ be a closed interval of length less than $1 / j$ in which $I_{3} \backslash G_{j}$ is categorically dense. Applying the above claim to this interval $H$ yields a contradiction.

Now, since each $G_{j}$ is residual in $I_{3}$, so is $G \equiv \bigcap_{j=1}^{\infty} G_{j}$. Let $x \in S(f) \cap$ $f^{-1}(J) \cap G$, which is nonempty since $G$ and $f^{-1}(J)$ are residual in $I_{3}$ and $S(f)$ is second category in $I_{3}$. We have that for each $j$, there is a $0<h_{j}<1 / j$ such that one of $x+h_{j}, x-h_{j}$ belongs to $f^{-1}(K)$ while the other belongs to $f^{-1}(J)$. Without loss of generality, suppose that $x+h_{j} \in f^{-1}(K)$. Then

$$
\begin{aligned}
\left|f\left(x+h_{j}\right)+f\left(x-h_{j}\right)-2 f(x)\right| & \geq\left|f\left(x+h_{j}\right)-f(x)\right|-\left|f\left(x-h_{j}\right)-f(x)\right| \\
& >2 \varepsilon / 3-\varepsilon / 3=\varepsilon / 3,
\end{aligned}
$$

which contradicts the assumption that $x \in S(f)$ and completes the proof.

Corollary 2. If $f: R \rightarrow R$ has the Baire property in the wide sense, then $S(f)$ has the Baire property in the wide sense.

Theorem 3. If $f: R \rightarrow R$ is Lebesgue measurable, then $S(f) \backslash C(f)$ has measure zero.

Proof. Suppose that $S(f) \backslash C(f)$ has positive outer measure. Then there exists a perfect set $P$ with positive measure such that $S(f) \backslash C(f)$ has full measure in 
$P$. We shall show that this situation is impossible by showing that $C(f)$ has full measure in this $P$.

Suppose that $C(f)$ does not have full measure in $P$ and $P_{1}$ is a perfect subset of $P$ such that $\mu\left(P_{1}\right)>0$ and $P_{1} \cap C(f)=\varnothing$. Since $P_{1} \cap C(f)=\varnothing$, there exists a perfect set $P_{2} \subseteq P_{1}$ and a rational number $\varepsilon>0$ such that $\mu\left(P_{2}\right)>0$ and $\operatorname{osc}(f, x)>\varepsilon$ for each $x \in P_{2}$.

Now let $\left\{J_{k}\right\}$ be an enumeration of all open intervals of length $\varepsilon / 3$ having rational end points. Since $f$ is Lebesgue measurable, there exists $k$ such that $f^{-1}\left(J_{k}\right) \cap P_{2}$ has positive measure. Let $P_{3} \subseteq P_{2}$ be a bounded perfect set such that $P_{3} \subseteq f^{-1}\left(J_{k}\right)$, and let $\mu\left(P_{3}\right)>3(d-c) / 4$, where $c$ and $d$ are the left end point and the right end point of $P_{3}$, respectively.

Let $J \equiv J_{k} \equiv(a, b)$ and $K \equiv R \backslash(a-2 \varepsilon / 3, b+2 \varepsilon / 3)$. For each natural number $j$, let $G_{j}=\left\{x \in P_{3}\right\}$ : there is a $0<h<1 / j$ such that one of $x+h, x-h$ belongs to $f^{-1}(K)$ while the other belongs to $\left.f^{-1}(J)\right\}$. Each $G_{j}$ has inner measure at least $3(d-c) / 8$. To see this, consider the following argument: Let $\left\{I_{t}\right\}_{j=1}^{n}$ be a partition of $[c, d]$ such that mesh of $\left\{I_{t}\right\}_{j=1}^{n}<1 / j$. Let $A \equiv\left\{1 \leq m \leq n\right.$ : the interior of $I_{m}$ contains a point of $\left.P_{3}\right\}$. For each $m \in A$, let $q_{m} \in f^{-1}(K) \cap I_{m}$. We know that there are such $q_{m}$ because $\operatorname{osc}(f, x)>\varepsilon$ for each $x \in P_{3}$. Then $\left(q_{m}+\left(P_{3} \cap I_{m}\right)\right) / 2 \subseteq G_{j} \cap I_{m}$ and

$$
\begin{aligned}
\frac{3}{8}(d-c) & \leq \mu\left(P_{3}\right) / 2=\sum_{m \in A} \mu\left(P_{3} \cap I_{m}\right) / 2 \\
& =\sum_{m \in A} \mu\left(q_{m}+\left(P_{3} \cap I_{m}\right)\right) / 2 \\
& \leq \sum_{m \in A} \mu_{*}\left(G_{j} \cap I_{m}\right) \leq \mu_{*}\left(G_{j}\right) .
\end{aligned}
$$

Now let $G=\bigcap G_{j}$. Since for each $j, \mu_{*}\left(G_{j}\right)>3(d-c) / 8$, we have that $\mu_{*}(G)>3(d-c) / 8 . G \cap P_{3} \cap S(f) \neq \varnothing$ follows from the fact that $G \cap P_{3}$ has positive inner measure and $S(f)$ has full outer measure in $P$ and hence in $P_{3}$.

Let $x \in G \cap P_{3} \cap S(f)$. We have that for each $j$, there is a $0<h_{j}<1 / j$ such that one of $x+h_{j}, x-h_{j}$ belongs to $f^{-1}(K)$ while the other belongs to $f^{-1}(J)$. Without loss of generality, suppose that $x+h_{j} \in f^{-1}(K)$. Then

$$
\begin{aligned}
\left|f\left(x+h_{j}\right)+f\left(x-h_{j}\right)-2 f(x)\right| & \geq\left|f\left(x+h_{j}\right)-f(x)\right|-\left|f\left(x-h_{j}\right)-f(x)\right| \\
& >2 \varepsilon / 3-\varepsilon / 3=\varepsilon / 3,
\end{aligned}
$$

which contradicts the assumption that $x \in S(f)$ and completes the proof.

Corollary 4. If $f$ is Lebesgue measurable, then $S(f)$ is Lebesgue measurable.

In [2], under the assumption of the continuum hypothesis, Erdös constructed two groups, one of which was simultaneously measure zero and second category, while the other was simultaneously first category and nonmeasurable. Although Erdös did not use this terminology, one of his groups is a Lusin set and the other a Sierpinski set. Some small modifications of his theorems yield our Lemmas 5 and 6.

Lemma 5. Under the assumption of the continuum hypothesis, there exists a Lusin set $G \subseteq R$ which has the following properties:

(1) $G$ is a topological group, and 
(2) $G$ contains sets $N_{1}$ and $N_{2}$ such that $N_{1} \cap N_{2}=\varnothing, N_{1}$ and $N_{2}$ are categorically dense in $R$, and $N_{1} \cup N_{2}$ is linearly independent over $Q$, the set of rationals.

Lemma 6. Under the assumption of the continuum hypothesis, there exists a Sierpinski set $G \subseteq R$ which has the following properties:

(1) $G$ is a topological group, and

(2) $G$ contains sets $N_{1}$ and $N_{2}$ such that $N_{1} \cap N_{2}=\varnothing, N_{1}$ and $N_{2}$ have full outer measure in every measurable set, and $N_{1} \cup N_{2}$ is linearly independent over $Q$.

The following is a well-known theorem of Sierpinski and Zygmund, the proof of which may be found in [7].

Lemma 7. There exists a function $g: R \rightarrow R$ such that $g \mid M$ is not continuous for every set $M$ which has cardinality $c$.

Brown and Prikry, in [1], constructed two functions, one of which is universally measurable and has the property that its restriction to every second category set is not continuous, while the other is $B_{r}$ measurable and has the property that its restriction to every set with positive outer measure is not continuous. In some sense our examples are not as strong as theirs; however, ours have the additional property of being symmetric on large sets.

Example 8. Under the assumption of the continuum hypothesis, there exists a universally measurable function $f: R \rightarrow R$ which is symmetric on a second category set, yet $f \mid M$ is not continuous for every second category set $M$ which has the Baire property in the wide sense. Note that for such $f, S(f) \backslash C(f)$ is second category.

Proof. Let $G, N_{1}$, and $N_{2}$ be as in Lemma 5, and let $g: R \rightarrow R$ be as in Lemma 7.

Now we want to construct $f$ which is symmetric on $N_{2}$. Let $A_{0}=N_{1}$. For each positive integer $n$, let $A_{n}=\left(2 N_{2}-A_{n-1}\right) \backslash\left(\bigcup_{i=0}^{n-1} A_{i}\right)$. Let $x \in A_{n}$ for some $n$. Then $x$ has a unique representation in terms of $N_{1} \cup N_{2}$ since $N_{1} \cup N_{2}$ is linearly independent over $Q$. Let $p(x)$ be the only element of $N_{1}$ that appears in this representation of $x$. Now we define $f$ in the following manner: $f(x)=(-1)^{n} g(p(x))$ if $x \in A_{n}$ for some $n$, otherwise let $f(x)=0$. In particular, note that $f$ is zero on $N_{2}$.

Now we want to show that $f$ is symmetric on $N_{2}$. Let $h>0$ and $x \in N_{2}$. If both $x+h$ and $x-h$ belong to the complement of $\left(\bigcup_{i=0}^{\infty} A_{i}\right)$, then we are done. Therefore, let us assume that one of $x+h$ or $x-h$ belongs to some $A_{n}$. Without loss of generality, let us assume that $x+h \in A_{n}$. Then $h \in-x+A_{n} \Rightarrow-h \in x-A_{n} \Rightarrow x-h \in 2 x-A_{n}$. Note that $x-h \in 2 x-A_{n}$ implies that $x-h \in A_{n-1}$ or $x-h \in A_{n+1}$ and that $p(x-h)=p(x+h)$. So we have that

$$
f(x-h)=(-1)^{n} g(p(x-h))=(-1)^{n} g(p(x+h))=(-1) f(x+h) .
$$

Therefore, $|f(x+h)+f(x-h)-2 f(x)|=0$ for every $x \in N_{2}$ and $h \in R$.

That $f$ is universally measurable follows from the fact that $f$ is zero on the complement of the $U_{0}$ set $G$. ( $G$ is $U_{0}$ because $G$ is a Lusin set.) 
Let $M$ be a second category set which has the Baire property in the wide sense. Let $I$ be a closed interval such that $M$ contains a $G_{\delta}$ set which is dense in $I$. We want to show that $f \mid M$ is not continuous. To obtain a contradiction, assume that $f \mid M$ is continuous. Since $N_{1}$ is categorically dense in $R$ and $M$ contains a dense $G_{\delta}$ set subset of $I, M \cap N_{1}$ is second category. The cardinality of $M \cap N_{1}$ is $c$ because we are assuming the continuum hypothesis. This implies that $f\left|\left(M \cap N_{1}\right)=g\right|\left(M \cap N_{1}\right)$ is continuous, contradicting that $g$ is a Sierpinski-Zygmund function, and completes the proof.

Example 9. Under the assumption of the continuum hypothesis, there exists a $B_{r}$ function $f: R \rightarrow R$ which is symmetric on a set with positive outer measure, yet $f \mid M$ is not continuous for every positive measure set $M$. Note that for such $f, S(f) \backslash C(f)$ has positive outer measure.

Proof. Let $G, N_{1}$, and $N_{2}$ be as in Lemma 6, and let $g: R \rightarrow R$ be as in Lemma 7.

We now define $f$ exactly as in the previous example. It follows in the same manner that for every $x \in N_{2}$ and $h \in R$ we have

$$
|f(x+h)+f(x-h)-2 f(x)|=0 .
$$

That $f$ has the Baire property in the restricted sense follows from the fact that $f$ is zero on the complement of the AFC set $G .(G$ is AFC because $G$ is a Sierpinski set.)

Let $M$ be a set which has positive measure. We want to show that $f \mid M$ is not continuous. To obtain a contradiction, assume that $f \mid M$ is continuous. Since $N_{1}$ has the property that it has full outer measure in every set with positive outer measure, $M \cap N_{1}$ has positive outer measure. The cardinality of $M \cap N_{1}$ is $c$ because we are assuming the continuum hypothesis. This implies that $f\left|\left(M \cap N_{1}\right)=g\right|\left(M \cap N_{1}\right)$ is continuous, contradicting that $g$ is a Sierpinski-Zygmund function, and completes the proof.

Lemma 10. If $C$ is a Cantor set which is linearly independent over $Q$, then the group $G$ generated by $C$ over $Q$, i.e., the set of all finite linear combinations of $C$ with rational coefficients, is of the first category and measure zero.

Proof. Let $C$ be a Cantor set which is linearly independent over $Q$, and let $G$ be the group generated by $C$ over $Q$.

First we will give a proof assuming that $C$ is not a maximal linearly independent set over $Q$. Consider $M \equiv r_{1} C+r_{2} C+\cdots+r_{n} C$ where $r_{1}, r_{2}, \ldots, r_{n}$ are rational numbers. $M$ is a compact set, and since $C$ is not maximal, $M$ has to be of the first category, for otherwise $M-M$ would contain an interval and the rational multiples of $M-M$ would cover the line, contradicting that $C$ is not maximal. $M$ also has measure zero, for otherwise $M-M$ would contain an interval and the rational multiples of $M-M$ would cover the line, contradicting that $C$ is not maximal. $G$ is the countable union of such $M$ 's. Therefore $G$ is first category and measure zero.

Now let us consider the general case. $C$ can be written as the union of a singleton set $\{p\}$ and a countable collection of Cantor sets $\left\{F_{k}\right\}$, where $F_{k+1} \supseteq F_{k}$ for each $k$ and none of the $F_{k}$ 's contain $p$. Let $G_{k}$ be the group generated by $F_{k}$ over $Q$ and let $H=\bigcup G_{k}$. Each of the $G_{k}$ is of the first category and measure zero because $F_{k}$ is not maximal. Since $H$ is the countable 
union of first category measure zero sets, $H$ is of the first category and measure zero. Note that $G=\bigcup_{r \in Q} H+r p$. Therefore, $G$ is of the first category and measure zero.

Theorem 11. There are $2^{c}$ many functions $f: R \rightarrow R$ such that

(1) $f$ is Lebesgue measurable,

(2) $f$ has the Baire property in the wide sense,

(3) $S(f) \backslash C(f)$ has cardinality $c$.

Proof. Let $C$ be a Cantor set such that $C$ is linearly independent over $Q$. The existence of such a set follows from a result of von Neumann [6].

Let $D$ be a countable dense subset of $C$. For each $K \subseteq C \backslash D$, we define $f_{K}: R \rightarrow R$ in the following fashion: Let $A_{0}=D$. For each positive integer $n$, let $A_{n}=\left(2 K-A_{n-1}\right) \backslash\left(\bigcup_{i=0}^{n-1} A_{i}\right)$. Let $x \in A_{n}$ for some $n$. Each $x \in A_{n}$ has a unique representation in terms of $K \cup D$ because $K \cup D$ is linearly independent over $Q$. Now let $f_{K}(x)=1$ if $x \in A_{n}$ for some even $n, f_{K}(x)=-1$ if $x \in A_{n}$ for some odd $n$, and $f_{K}(x)=0$ otherwise. Note that $f$ is zero on $K$ and $G^{c}$, where $G$ is the group generated by finite linear combinations of $C$ with rational coefficients.

Now we want to show that $f$ is symmetric on $K$. Let $h>0$ and $x \in K$. If both $x+h$ and $x-h$ belong to the complement of $\left(\bigcup_{i=0}^{\infty} A_{i}\right)$, then we are done. Therefore, let us assume that one of $x+h$ or $x-h$ belongs to some $A_{n}$. Without loss of generality, let us assume that $x+h \in A_{n}$. Then $h \in-x+A_{n} \Rightarrow-h \in x-A_{n} \Rightarrow x-h \in 2 x-A_{n}$. Since each $x \in A_{n}$ has a unique representation in terms of $K \cup D$ and $x-h \in 2 x-A_{n}$, we have that $x-h \in A_{n-1}$ or $x-h \in A_{n+1}$. Therefore, $\left|f_{K}(x+h)+f_{K}(x-h)-2 f_{K}(x)\right|=0$ for every $x \in K$ and $h \in R$.

$G$ is of the first category and measure zero by Lemma 10. Each $f_{K}$ is Lebesgue measurable and has the Baire property in the wide sense because $f_{K}$ is zero on the complement of $G$.

The last thing that we need to show is that if $K, L \subseteq C \backslash D$ and $K \neq L$, then $f_{K} \neq f_{L}$. To see this, consider $x \in K \backslash L$ or $x \in L \backslash K$ and $d \in D$. Then one of $f_{K}(2 x-d)$ and $f_{L}(2 x-d)$ is zero while the other is 1 or -1 .

Since there are $2^{c}$ many sets $K \subseteq C \backslash D$ which have cardinality $c$, the collection of all functions that satisfy properties $(1)-(3)$ of our theorem has cardinality $2^{c}$.

\section{ACKNOWLEDGMENT}

The author would like to thank Professor Mike Evans for raising questions that led to some of the results in this paper.

\section{REFERENCES}

1. J. B. Brown and K. Prikry, Variations on Lusin's theorem, Trans. Amer. Math. Soc. $\mathbf{3 0 2}$ (1987), 77-86.

2. P. Erdös, Some remarks on subgroups of real numbers, Colloq. Math. 42 (1979), 119-120.

3. M. J. Evans and L. Larson, The continuity of symmetric and smooth functions, Acta Math. Hungar. 43 (1984), 251-257.

4. A. Miller, Special subsets of the line, Handbook of Set Theoretic Topology (K. Kunen and J. Vaughn, eds.), North-Holland, Amsterdam, 1984. 
5. C. J. Neugebauer, Symmetric continuous and smooth functions, Duke Math. J. 31 (1964), 23-32.

6. J. von Neumann, Ein System algebraisch unabhangiger Zahlen, Math. Ann. 99 (1928), 134-141.

7. W. Sierpinski and A. Zygmund, Sur une fonction qui est discontinue sur tout ensemble de puissance du continu, Fund. Math. 4 (1923), 316-318.

8. E. M. Stein and A. Zygmund, On the differentiability of functions, Studia Math. 23 (1964), 247-283.

Department of Mathematics, North Carolina State University, Raleigh, North CarOLINA 27695-8205

E-mail address: UDARJI@GUEST1.MATH.NCSU.EDU 